\title{
Frakturendoprothetik am Ellbogengelenk
}

\author{
Michael Plecko
}

\section{Zusammenfassung}

Dislozierte instabile Frakturen am Ellbogengelenk werden i.d.R. operativ versorgt. Wenn es die Frakturform und die Knochenqualität zulassen, wird meist eine Osteosynthese mit Schrauben und Platten durchgeführt. Eine Osteosynthese führt aber nur dann zu einem guten funktionellen Ergebnis, wenn die erzielte Stabilität eine frühfunktionelle Nachbehandlung ermöglicht. Bei Frakturen mit Trümmerzonen stellt, insbesondere beim betagten Patienten mit Osteoporose, die primäre Frakturendoprothese eine gute Behandlungsalternative dar. Hier zeigt am distalen Humerus die Datenlage Vorteile für ein primär prothetisches Vorgehen. Die zementierten teilverblockten Ellbogentotalendoprothesen zeichnen sich in dieser Patientengruppe durch eine frühe Gebrauchsfähigkeit des Armes und gute Langzeitergebnisse aus. Es ist aber derzeit noch von einer begrenzten Belastbarkeit auszugehen. Am Radiuskopf ist bei nicht stabil rekonstruierbaren Mehrfrag- mentfrakturen der prothetische Ersatz indiziert, da meist ligamentäre und ossäre Begleitverletzungen vorliegen. Die Wiederherstellung der radialen Abstützung ist in diesen Fällen für eine balancierte Kraftübertragung und eine ausreichende Stabilität des Ellbogengelenks erforderlich. Nur in jenen seltenen Fällen, in denen bei geriatrischen Patienten mit komplexer Radiuskopffraktur keine Bandverletzung oder Verletzung der Membrana interossea vorliegt, kann eine Radiuskopfresektion ebenfalls zu guten Ergebnissen führen.

\section{Fracture Endoprosthesis on the Elbow Joint}

Most displaced unstable fractures of the elbow need surgical treatment. The preferred method is to use anatomical reconstruction and stabilisation with screws and plates, in so far as this is feasible with the individual fracture pattern and bone quality. Good clinical results may only be obtained if stability allows early movement and functional after-treatment. In elderly patients with complex fracture pattern and poor bone quality due to osteoporosis, primary prosthetic replacement is an excellent alternative. There is some evidence that primary arthroplasty for comminuted fractures in elderly people is advantageous. Semiconstrained total elbow prosthesis fixed by cement usually leads to early restoration of function and good long-term results. Nevertheless some restrictions to weight lifting must be accepted. Radial head prostheses are indicated in patients with nonreconstructable, multiple fragmentation of the radial head, as there are concomitant ligamentous or bony injuries in most of these cases. In these cases, reconstruction of the radial column is necessary to achieve balanced force transmission over the elbow joint, as well as sufficient stability. In orthogeriatric patients with complex fractures of the radial head but absence of ligamentous injuries or rupture of the interosseous membrane, resection of the radial head may also lead to good results.

\section{Einleitung}

Die Endoprothetik am Ellbogengelenk hat in den letzten 10 Jahren zunehmend mehr Beachtung gefunden. Sie kommt inzwischen nicht nur bei fortgeschrittenen degenerativen Veränderungen des Gelenks (Arthrose, Arthritis, Gelenkzerstörung bei Hämophilie) und als Rettungsoperation nach fehlgeschlagenen Ersteingriffen (fehlgeschlagenen Frakturrekonstruktionen, chronische Instabilität nach fehlgeschlagenem Bandersatz, dekompensierten Pseudarthrosen und Osteonekrosen nach Frakturversorgung) zum

OP-JOURNAL 2016; 32: 182-189

(c) Georg Thieme Verlag KG Stuttgart · New York DOI http://dx.doi.org/10.1055/s-0042-121504
Einsatz, sondern wird auch vermehrt zur primären Frakturversorgung besonders beim älteren Patienten diskutiert. Hier sind in erster Linie die komplexen, nicht stabil rekonstruierbaren, distalen Humerusfrakturen zu nennen, die besonders bei vorliegender Osteoporose eine große Herausforderung in der Behandlung darstellen. Nach anfänglich ernüchternden Ergebnissen in früheren Jahren durch die Verwendung von gekoppelten Endoprothesen mit sehr hohen frühzeitigen Lockerungs- und Komplikationsraten, sind seit der Einführung von modularen Systemen und teilgekoppelten Prothesen deutlich bessere Ergebnisse erzielt worden.

Neben der Totalendoprothese spielt am Ellbogengelenk auch der prothetische Ersatz des Radiuskopfs eine nicht un- erhebliche Rolle. Insbesondere nicht stabil rekonstruierbare Trümmerfrakturen, meist in Kombination mit einer komplexen Bandverletzung bzw. Instabilität, sind eine häufige Indikation für eine Radiuskopfprothese. In den letzten Jahren wurde das Spektrum der Endoprothetik am Ellbogengelenk durch den Ersatz des Capitulums am distalen Humerus ergänzt, wodurch ein kompletter Ersatz des radialen Gelenkabschnitts (Radiuskopf und radialer Anteil der Humerusgelenkfläche) möglich wurde.

\section{Frakturprothetik der distalen Humerusfraktur}

Distale Humerusfrakturen machen mit einer Inzidenz von 5,7 Fällen auf 100000 Personen pro Jahr ungefähr 2\% aller 
Frakturen aus [1]. Besonders beim älteren Patienten mit Osteoporose führen oft schon einfache Stürze zu sehr komplexen, weit distal gelegenen Frakturen. Dies macht eine stabile osteosynthetische Versorgung oft schwierig bis unmöglich. Die Versorgung mit Platten, heute meist winkelstabil und vorgeformt, stellt nach wie vor den „Goldstandard“ in der Versorgung distaler Humerusfrakturen dar [2-4], ist jedoch besonders beim älteren Patienten mit einer hohen Komplikationsrate (bis zu 30\%) behaftet [5,6]. Rauchen, Übergewicht, Diabetes mellitus und vorbestehende Arthrose wurden als Risikofaktoren für ein Implantatversagen bzw. eine Pseudarthrosebildung identifiziert [7]. In aktuelleren Studien mit modernen Implantaten und einem parallelen Plattenkonzept konnten jedoch die Ergebnisse deutlich verbessert werden [6]. Eine stabile anatomische Rekonstruktion des Gelenkblocks und eine ausreichende Stabilität für eine frühfunktionelle Nachbehandlung sind ausschlaggebend für den Erfolg der Osteosynthese.

Besonders beim alten Patienten gibt es jedoch Frakturformen, die mit offener Rekonstruktion und Plattenosteosynthese nicht sinnvoll versorgbar sind. Daher wurde für ausgewählte Patienten nach alternativen Behandlungsverfahren gesucht. Die Ellbogengelenkprothese stellt für ältere Patienten mit Osteoporose und mit komplexen distalen Humerusfrakturen eine gute Alternative zur Osteosynthese dar. In ersten Serien konnten ermutigende Ergebnisse erzielt werden [8]. Seither hat die Ellbogengelenkprothetik deutlich an Popularität gewonnen, und in einer Vielzahl von Studien konnte ihre hohe Wertigkeit in der Versorgung von Problemfrakturen am distalen Humerus bestätigt werden [9-15]. In sämtlichen Studien konnte ein gutes oder sehr gutes funktionelles Ergebnis nach einem allgemein akzeptierten validen Scoresystem (MEPS = Mayo Elbow Performance Score) erreicht werden. Neben diesen Fallserien wurde inzwischen auch eine prospektive randomisierte direkte Vergleichsstudie zwischen Plattenosteosynthese und primärer Totalendoprothese durchgeführt und publiziert [16]. In dieser konnte eine Überlegenheit der primären Ellbogengelenktotalendoprothese gegenüber der Plattenosteosynthese bei komplexen intraartikulären Frakturen am distalen Humerus bei Patienten über 65 Jahren dargestellt werden. Retrospektive Vergleichsstudien zeigten unterschiedliche Ergebnisse, wo-
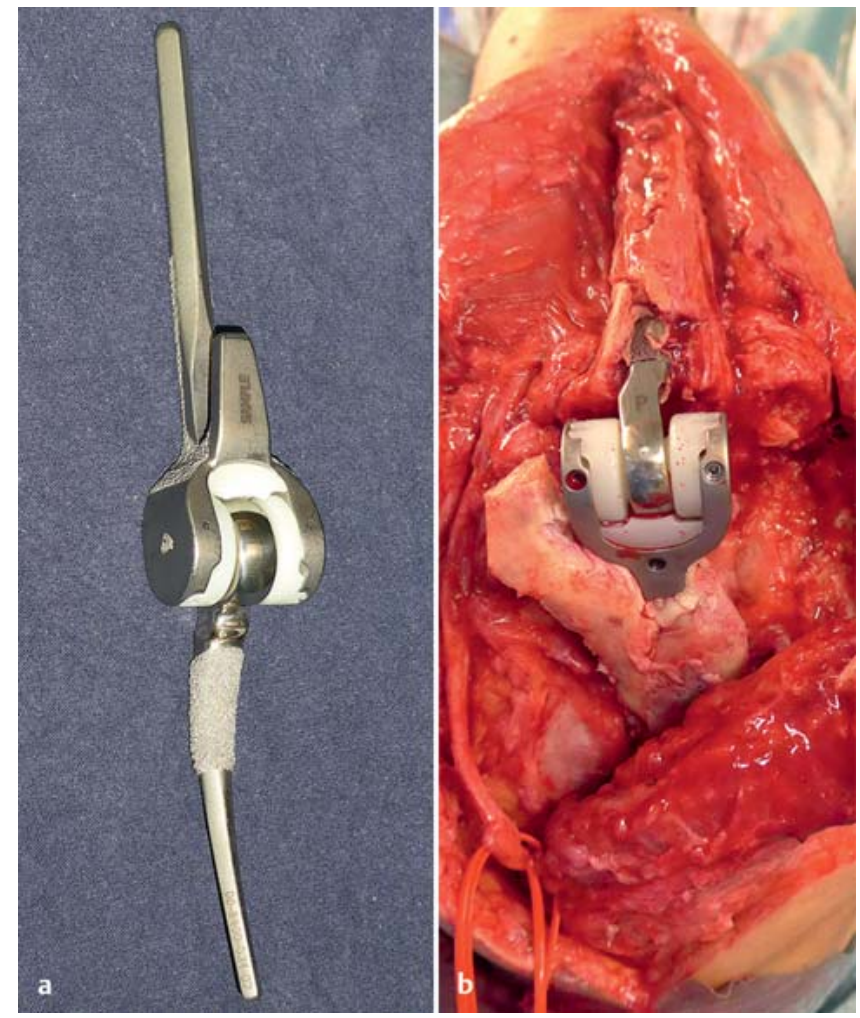

Abb. $1 \mathrm{a}$ und $\mathrm{b}$ Teilverblockte Ellbogentotalendoprothese: a Implantat, b intraoperativer Situs.

bei bei manchen ein Vorteil für die primäre Frakturprothetik angegeben wurde, während andere wie die von Egol [17] im Gegensatz dazu keinen signifikanten Unterschied bestätigen konnten. Eine frühere Beweglichkeit, Schmerzfreiheit und eine raschere Gebrauchsfähigkeit werden mit einer lebenslang notwendigen eingeschränkten Belastbarkeit des Armes erkauft. Neuere Entwicklungen im Prothesendesign lassen jedoch hoffen, dass die Belastbarkeit der Ellbogengelenktotalendoprothesen zunehmen wird und dadurch die Standzeiten der Prothesen auch bei Patienten mit geringerer Compliance deutlich verbessert werden könnten.

Der Einsatz von modularen, teilgekoppelten Ellbogengelenktotalendoprothesen stellt bei komplexen intraartikulären Frakturen des distalen Humerus eine sehr gute Versorgungsoption dar. Eine rasche Wiederherstellung der Alltagsfunktion steht einer reduzierten Belastbarkeit gegenüber.

\section{Operative Technik}

Für die Versorgung der komplexen distalen Humerusfraktur beim alten Patienten wird in erster Linie ein teilverblockter Prothesentyp in zementierter Form verwendet. Unverblockte Prothesentypen sowie Hemiprothesen bleiben bestimmten Frakturformen vorbehalten, bei denen sowohl der radiale als auch der ulnare Pfeiler intakt geblieben oder stabil rekonstruierbar sind, und bei denen auch die ligamentäre Stabilität wiederhergestellt werden kann. Da dies bei den Altersfrakturen meist nicht gegeben ist und diese Prothesentypen auch in der Nachbehandlung wesentlich mehr Mitarbeit des Patienten erfordern, bevorzugen wir beim geriatrischen Patienten i.d.R. einen teilverblockten zementierten Prothesentyp (Abb. 1). Hier lässt ein verbesserter Koppelungsmechanismus mit verbesserten Polyethylenteilen eine geringere Empfindlichkeit gegenüber Belastung und ein verbessertes Abriebverhalten erwarten.

Die Erfahrungen mit der Hemiprothese am Ellbogen des betagten Patienten sind in der Literatur kontrovers. Während einige Studien von positiven Erfahrungen berichten [18-20], berichten andere Autoren über ein Streckdefizit, Schmerzen bei Alltagsfunktionen und hohen Revisionsraten [21]. Auch Steinmann gibt als relative Kontraindikation für die Implantation einer Hemiprothese den geriatrischen hochbetagten Patienten an [22]. In unserem eigenen Vorgehen kommt die Hemiprothese bei komplexen nicht rekonstruierbaren Abscherfrakturen des distalen Humerus zur Anwendung sowie bei ausgedehnter Nekrose oder ausbleibender Heilung von Abscherfragmenten, wenn es sich um Patienten handelt, die 


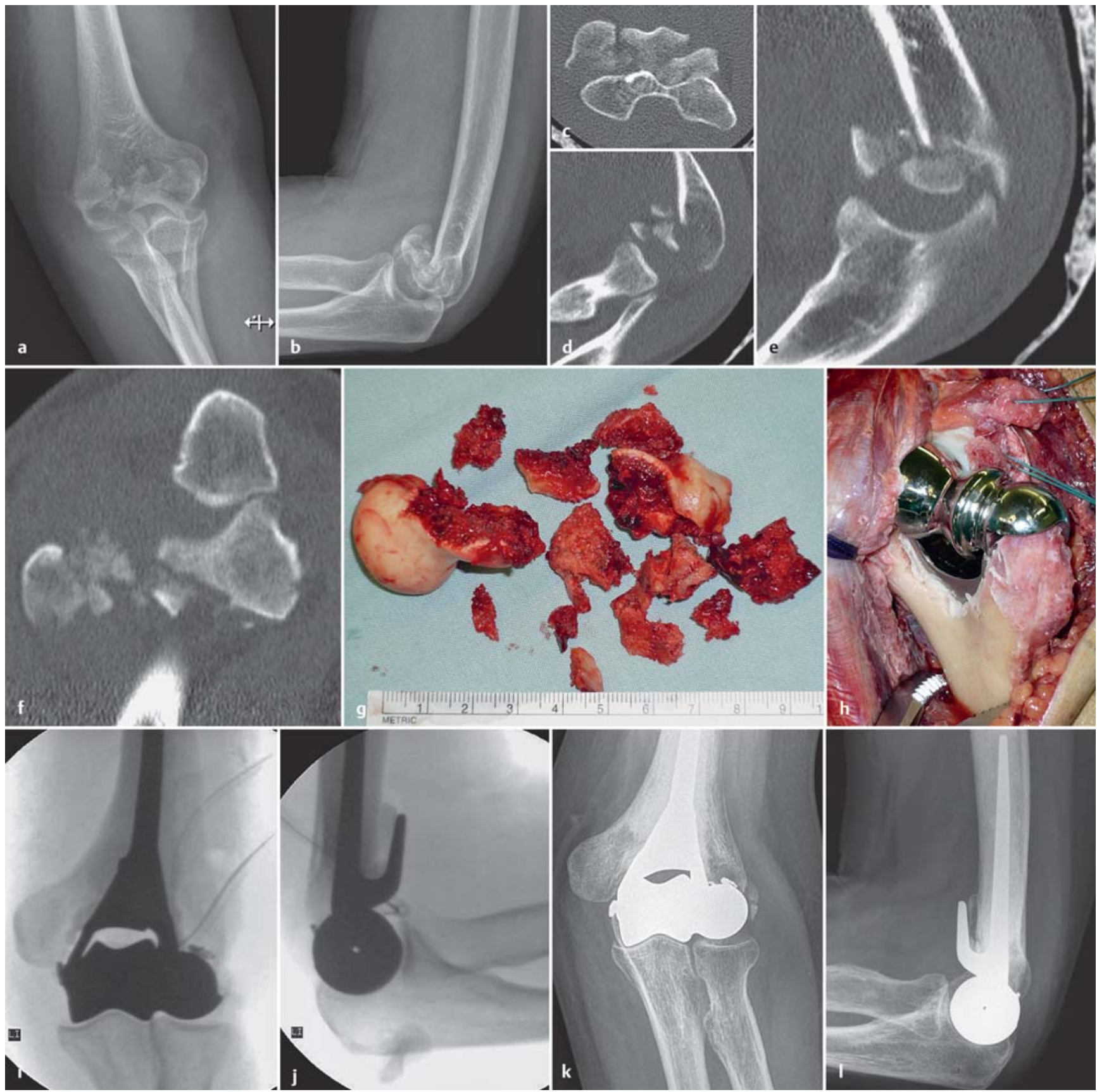

Abb. 2 a bis I 63-jährige Patientin. Sturz auf den linken Arm. Komplexe Gelenkfraktur des distalen Humerus. Versorgt mit einer zementierten Hemiprothese am distalen Humerus. a, b Nativröntgen a.-p. und seitlich; c-f CT-Schnitte; $\mathbf{g}$ entfernte Fragmente der Gelenkrolle; $\mathbf{h}$ intraoperativer Situs; $\mathbf{i}-\mathbf{j}$ intraoperative Bildwandlerkontrolle; $\mathbf{k}-\mathbf{l}$ postoperative Röntgenbilder nach 6 Monaten a.-p. und seitlich.

für eine Totalendoprothese zu jung sind und bei denen kein anderes rekonstruktives Verfahren mehr infrage kommt. Damit soll Zeit gewonnen werden, um nötigenfalls die Prothese später auf eine teilverblockte Totalendoprothese umbauen zu können (Abb. 2).

Die Implantation von Ellbogengelenktotalendoprothesen führen wir in Rückenlage des Patienten durch. Der betroffene Arm wird beweglich steril abgedeckt und auf einem von der Gegenseite über den Patienten geschobenen abgepolsterten Instrumententisch gelagert. In der Regel kommt eine sterile Oberarmblutsperre zur Anwendung. Wir bevorzugen eine gerade dorsale Hautinzision, die etwas seitlich (radial oder ulnar) an der Olecranonspitze vorbeizieht. Wenn immer möglich, wird bei Frakturen ein Zugang unter Belassen des Trizeps bzw. des Streckapparats angewandt. Meist kommt der von Studer et al. 2013 beschriebene laterale „Paraolekranon-Zugang“ zur Anwendung [23]. Der Vorteil liegt in dem Umstand, dass der Streckapparat nicht in der postoperativen Phase geschont werden muss und umgehend mit einer aktiven Bewegungstherapie begonnen werden kann (Abb. 3). Eine Alternative, bei welcher der Trizeps ebenfalls erhalten wird, ist der Zugang nach Pierce und Herndon. McKee konnte zeigen, dass eine Resektion der frakturierten Kondylen am Humerus zu keiner Beeinträchtigung des klinischen Ergebnisses bei einer teilverblockten Ellbogentotalendoprothese führt [24]. 


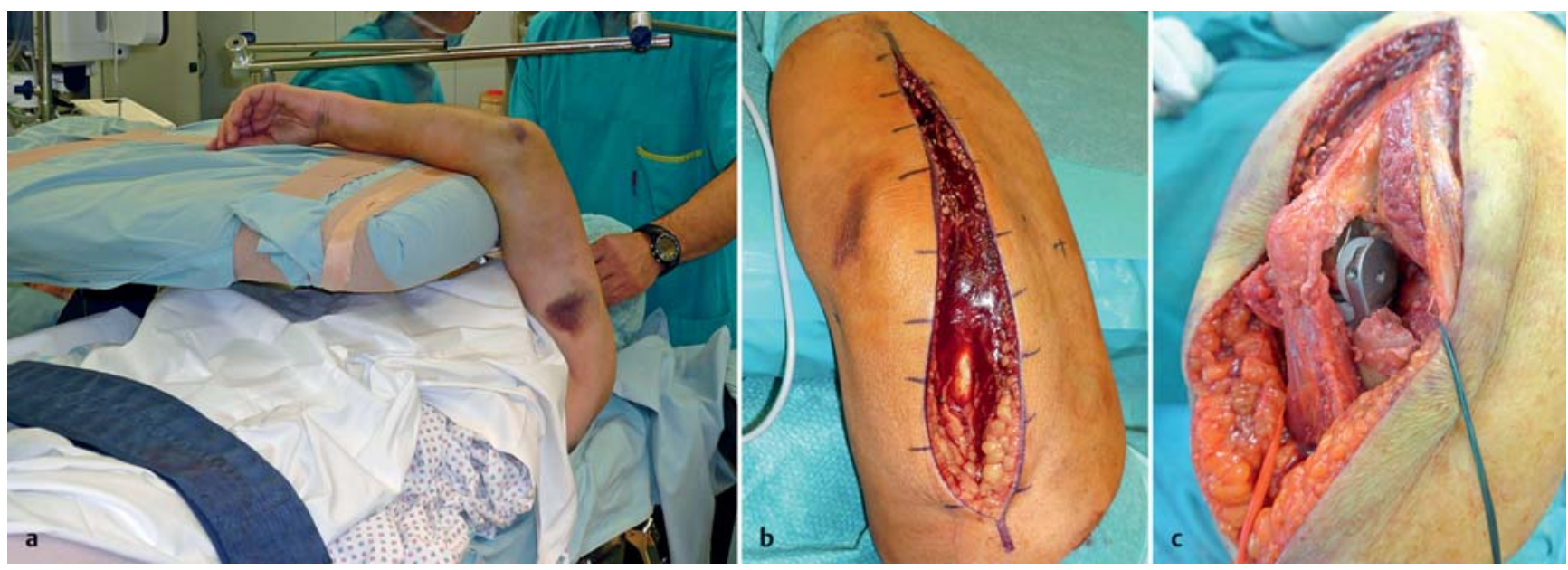

Abb. 3 a bis c Lagerung und operativer Zugang. a Patientin in Rückenlage, Arm auf gepolstertem Instrumententisch; b steril abgedeckt; dorsale gerade Hautinzision; c Paraolekranon-Zugang. Streckapparat erhalten.

Die zementierte teilverblockte Ellbogentotalendoprothese letzter Generation stellt bei komplexen, nicht sinnvoll rekonstruierbaren Frakturen des geriatrischen Patienten das bevorzugte Implantat dar und wird in der Fraktursituation über einen den Streckapparat erhaltenden Zugang implantiert.

Postoperativ wird der Arm für 24-48 Stunden in einer Schiene in Streckstellung ca. $45^{\circ}$ eleviert gelagert. Danach wird mit Bewegungsübungen begonnen, die je nach Weichteilbefund limitiert sind oder frei ohne Beschränkung durchgeführt werden können.

Bei den unverblockten Totalendoprothesen oder bei den Hemiprothesen muss eine Entlastung der Seitenbänder in einer dynamischen Schiene bis zu deren stabiler Heilung mindestens aber für 6 Wochen nach der Operation erfolgen.

Bei den teilverblockten Ellbogentotalendoprothesen wird bei uns nach wie vor eine Belastungsbeschränkung des Ellbogengelenks mit max. $5 \mathrm{~kg}$ verordnet, obwohl sich geriatrische Patienten meist nicht streng daran halten können. Wir haben aber dadurch im eigenen Krankengut noch keine frühzeitigen Lockerungen hinnehmen müssen. Die neuen Kopplungsmechanismen scheinen eine stärkere Belastung zuzulassen, obwohl hierzu valide Grenzwerte fehlen.

\section{Ergebnisse}

Die Evidenzlage bez. der Versorgung distaler Humerusfrakturen beim geriatrischen Patienten ist dürftig. Eine einzige prospektiv-randomisierte Studie zeigt signifikante Vorteile für den protheti- schen Ersatz im Vergleich zur Plattenosteosynthese [16]. In einer Metaanalyse konnte kein signifikanter Unterschied, jedoch ein deutlicher Trend zugunsten der Ellbogentotalendoprothese festgestellt werden [25]. Neben einem etwas besseren funktionellen Ergebnis ist es besonders die geringere Komplikationsrate, die bei ausgewählten Patienten für den prothetischen Ersatz spricht (Abb. 4). Der durchschnittliche Funktionsscore (MEPS) liegt zwischen 73 und 96, was einem guten und sehr guten Ergebnis entspricht [14]. Während die Beugung i.d.R. sehr gut möglich ist, weisen die meisten Patienten doch ein leichtes Streckdefizit (zwischen 10 und $33^{\circ}$ ) auf. Während in der Literatur eine 5-JahresÜberlebensrate der Prothese zwischen 72 und $84 \%$ und eine Komplikationsrate von 9,5\% angegeben wird, musste beim eigenen Krankengut bei Verwendung der Coonrad-Morrey-Ellbogentotalendoprothese nur in 2 Fällen, bei Verwendung der Latitude-Ellbogentotalendoprothese nur in einem Fall und bei Verwendung der Nexel-Ellbogentotalendoprothese mit dem verbesserten Prothesendesign in keinem einzigen Fall eine revisionspflichtige Komplikation hingenommen werden. Die primäre Anwendung der Ellbogentotalendoprothese zur Frakturversorgung führt im Vergleich zur Implantation nach fehlgeschlagener Osteosynthese $\mathrm{zu}$ einem tendenziell besseren funktionellen Ergebnis. Die Rate der relevanten Komplikationen, Revisionen und des notwendigen Prothesenausbaus ist aber bei den sekundär implantierten Ellbogentotalendoprothesen deutlich höher $[14,26]$.

\section{Die Radiuskopfprothese}

Der Radiuskopf ist ein wichtiger Stabilisator des Ellbogengelenks bei Valgusstress, insbesondere bei gleichzeitiger Verletzung des medialen Seitenbands. Bis zu 60\% des Lasttransfers können am Ellbogengelenk über das Gelenk zwischen Radiuskopf und Capitulum radialis humeri übertragen werden. Die Radiuskopffraktur ist die häufigste Fraktur am Ellbogengelenk. Bei schwereren Traumen kommt es häufig neben komplexen dislozierten Frakturformen zu zusätzlichen ligamentären Verletzungen, Frakturen an der Elle und Verletzungen der Membrana interossea. Während einfache, nicht oder wenig dislozierte Frakturen (Verschiebung $\leq 2 \mathrm{~mm}=$ Typ Mason I) gut konservativ behandelt werden können, werden stabil rekonstruierbare Frakturen mit einer Dislokation von mehr als $2 \mathrm{~mm}$ (Typ Mason II) osteosynthetisch mit Schrauben und ggf. mit einer Platte versorgt. Wichtig ist dabei neben der stufenfreien Reposition eine stabile Fixation, um eine frühfunktionelle Nachbehandlung zu ermöglichen. Frakturen mit erheblichen Trümmerzonen, die nicht stabil rekonstruierbar sind, werden im eigenen Vorgehen mit einer Radiuskopfprothese versorgt. Mit zunehmender Komplexität der Radiuskopffraktur nimmt die Häufigkeit von Begleitverletzungen zu. So konnte bei Mason-II- und -III-Frakturen in 54\% eine Ruptur des medialen Seitenbands festgestellt werden, in $80 \%$ eine Ruptur des lateralen Seitenbands und in 50\% eine Ruptur beider Bandkomplexe [27]. In bis zu $10 \%$ sind Radiuskopffrakturen mit Verrenkungen des Ellbogengelenks kombiniert. Bei zusätzlichem Abbruch der Spitze des Processus coronoideus ulnae 


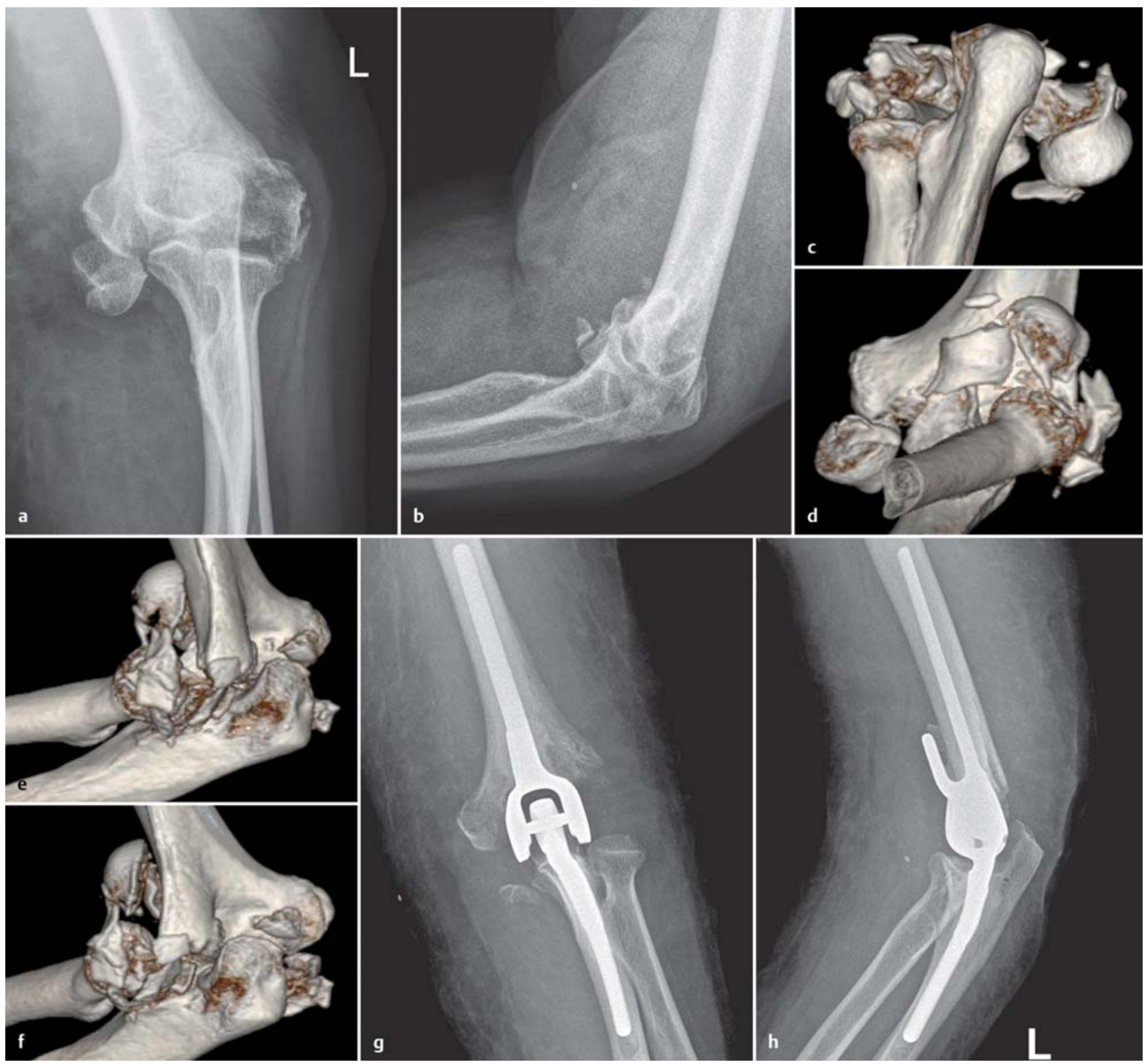

Abb. 4 a bis h 77-jährige Patientin. Sturz über den Teppich. Komplexe intraartikuläre Gelenkfraktur distaler Humerus und Abbruch vom Radiuskopf. a, b Nativröntgen a.-p. und seitlich; c-f 3-D-Rekonstruktion der CT-Schnitte; g, h postoperative Röntgenbilder a.-p. und seitlich. Zementierte teilverblockte Ellbogentotalendoprothese.

und Ruptur des Seitenbandapparats liegt eine sehr instabile Situation vor (= ,Terrible Triad“, Abb. 5). In diesen Fällen und in Fällen mit begleitender Ruptur der Membrana Interossea (= Essex-LoprestiVerletzung) ist unbedingt eine Rekonstruktion oder ein mechanisch kompetenter prothetischer Ersatz des Radiuskopfs erforderlich. Obwohl wir bei Radiuskopffrakturen i.d.R. eine CT-Untersuchung zur exakteren Beurteilung durchführen, kann die sinnhafte Rekonstruierbarkeit oft erst intraoperativ endgültig beurteilt werden. So ist bei der Versorgung komplexerer Frakturen eine Radiuskopfprothese stets vorbesprochen, sodass ein Umstieg von der Rekon- struktion auf die Endoprothese jederzeit möglich ist. Eine reine Radiuskopfresektion ohne Ersatz wird nur bei sehr betagten Patienten durchgeführt, bei denen weder eine Luxation oder Subluxation noch eine relevante ligamentäre Begleitverletzung oder Membranverletzung vorliegt.

Der prothetische Ersatz des Radiuskopfs ist bei komplexen, nicht stabil rekonstruierbaren Radiuskopffrakturen indiziert, bei denen eine zusätzliche ligamentäre Verletzung, knöcherne Verletzung oder eine Verletzung der Membrana interossea vorliegt.
Bevorzugt werden heute modulare Prothesensysteme, die ein Anpassen an die anatomischen Gegebenheiten erlauben (Abb 6). Die Prothesen sind i.d.R. aus Metall oder poliertem Carbon oder hochwertigem Polyethylen. Silikonprothesen werden aufgrund der mangelnden mechanischen Kompetenz nicht mehr verwendet. Grundsätzlich werden Kurzschaft- von Langschaftprothesen unterschieden sowie Prothesen mit einem starren Kopf von solchen mit einem flexiblen Kopf (bipolare Prothesen). 


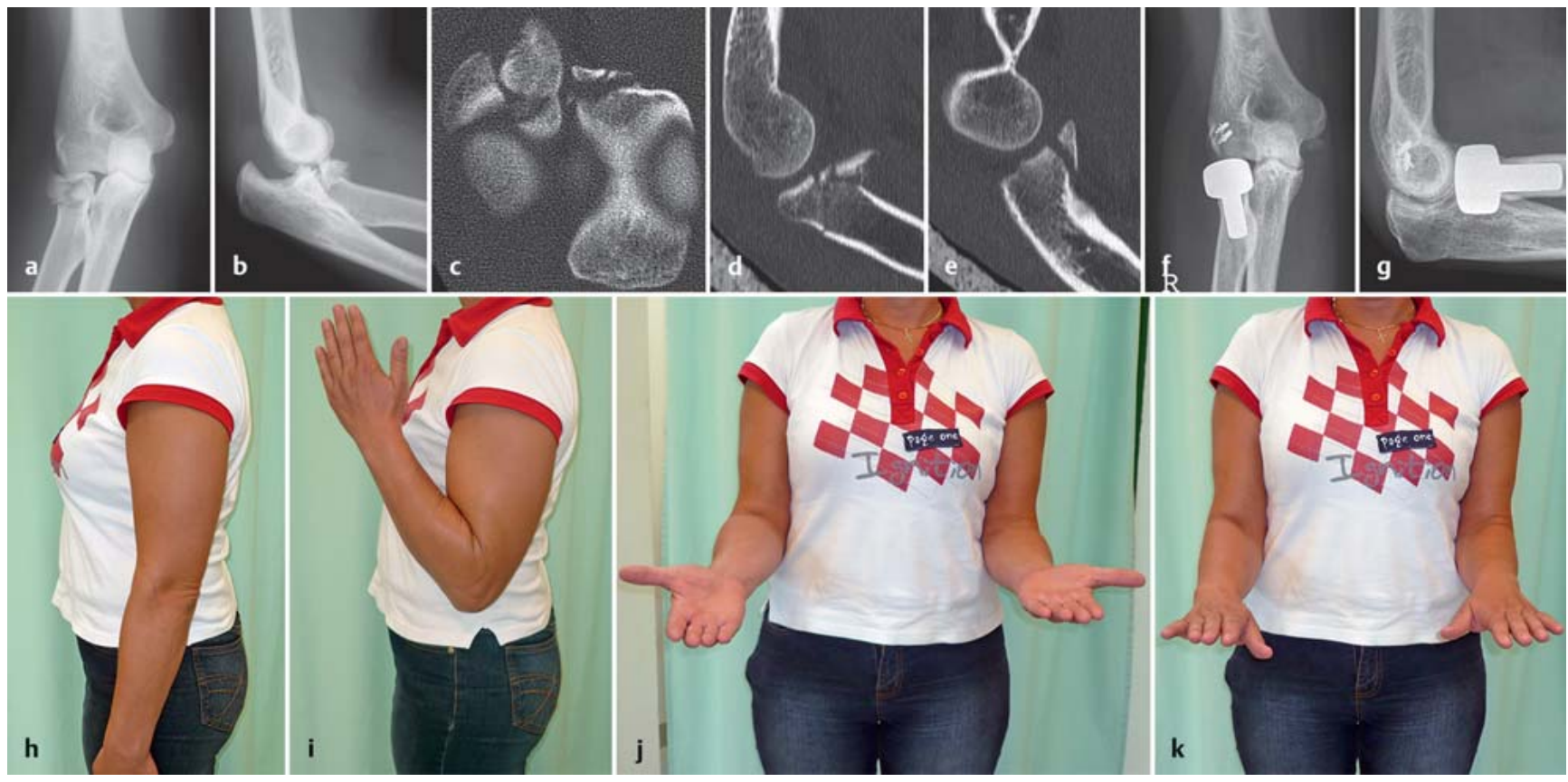

Abb. 5 a bis k 45-jährige Patientin. Sturz von einem Baum. Luxation des rechten Ellbogengelenks mit Radiuskopffraktur und Abbruch des Processus coronoideus der Elle (= „Terrible Triad“). a, b Nativröntgen a.-p. und seitlich; c-e CT-Schnitte; f-k Nativröntgen a.-p./seitlich und Funktion nach 5 Jahren.

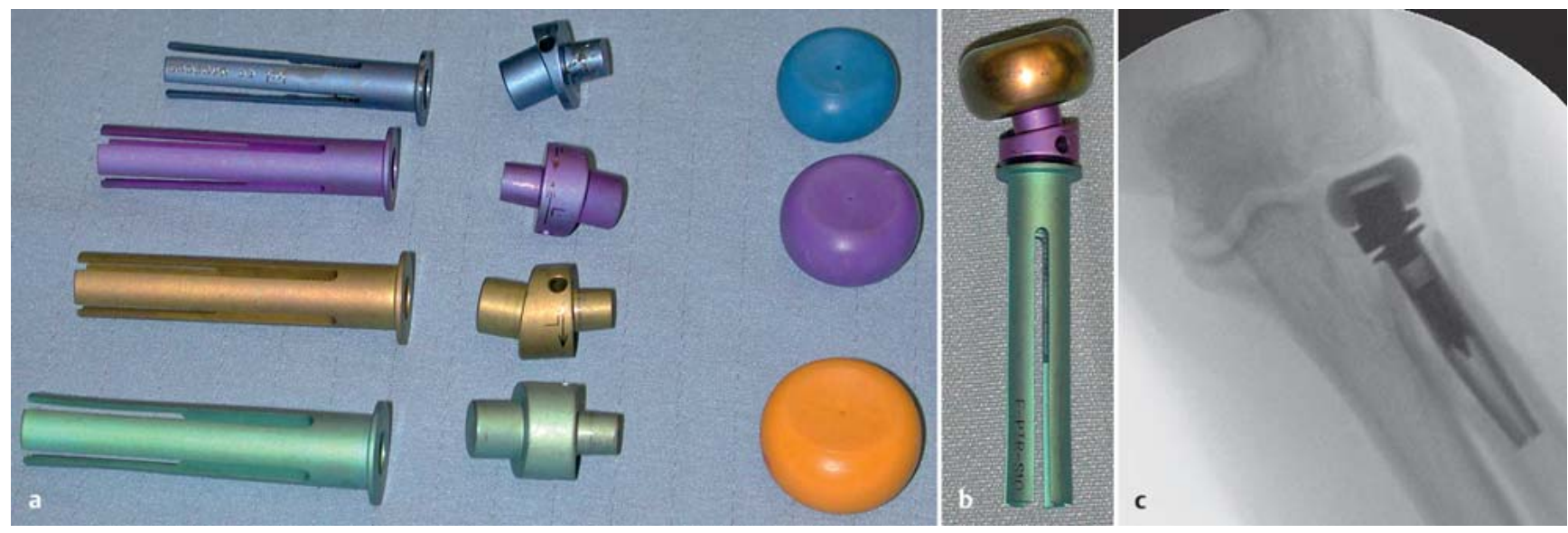

Abb. 6 a bis c Modulare Radiuskopfprothese. a verschiedene Schaftgrößen, Halslängen und Kopfdurchmesser; b zusammengestellte Probeprothese; $c$ Prothese in situ (intraoperatives Bildwandlerbild).

\section{Operative Technik}

Die Radiuskopfprothesen werden entweder zementiert oder zementfrei implantiert oder weisen einen polierten Schaft auf. Sie werden entweder über einen Kocher-Zugang, einen Kaplan-Zugang oder einen Extensor-digitorum-communisSplitting-Zugang eingebracht (Abb. 7). In der Fraktursituation sind manchmal Langschaftprothesen zur Überbrückung der Frakturzone erforderlich, wobei auf eine korrekte Rekonstruktion des Schaft-Hals-Winkels geachtet werden muss. Die Krümmung des proximalen Radius weist i.d.R. von der Tuberositas radii (Ansatz der distalen Bizepssehne) weg und zeigt in Richtung des Speichen-
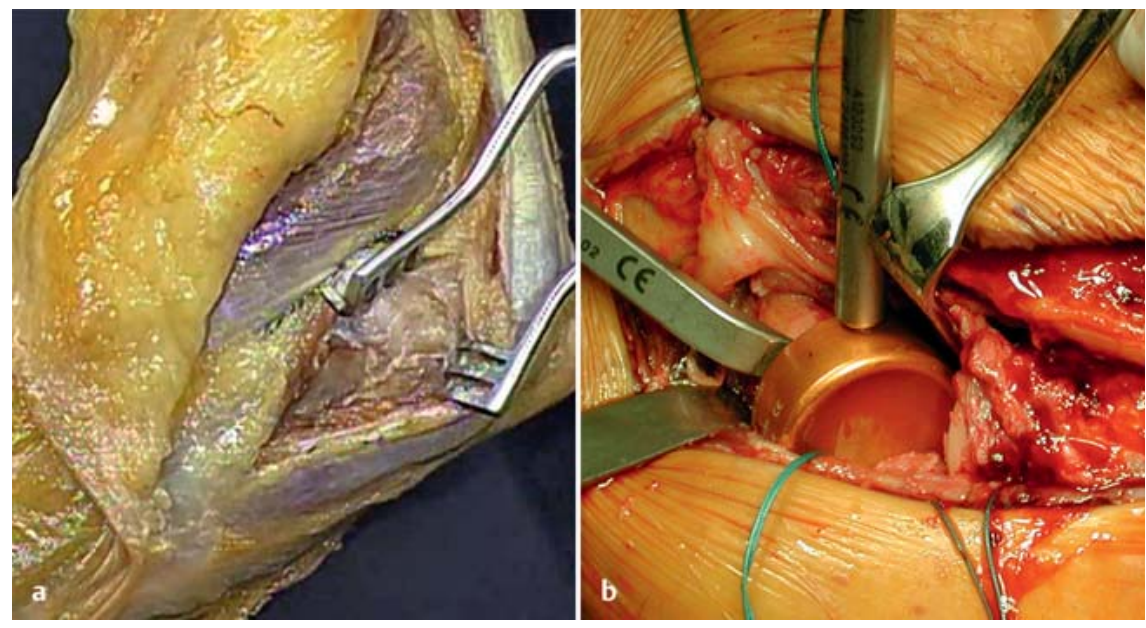

Abb. 7 a und b Operativer radialer Zugang: a Kocher-Intervall - zwischen M. anconeus* und M. extensor carpi ulnaris**; b intraoperativer Situs mit Probeprothese. 


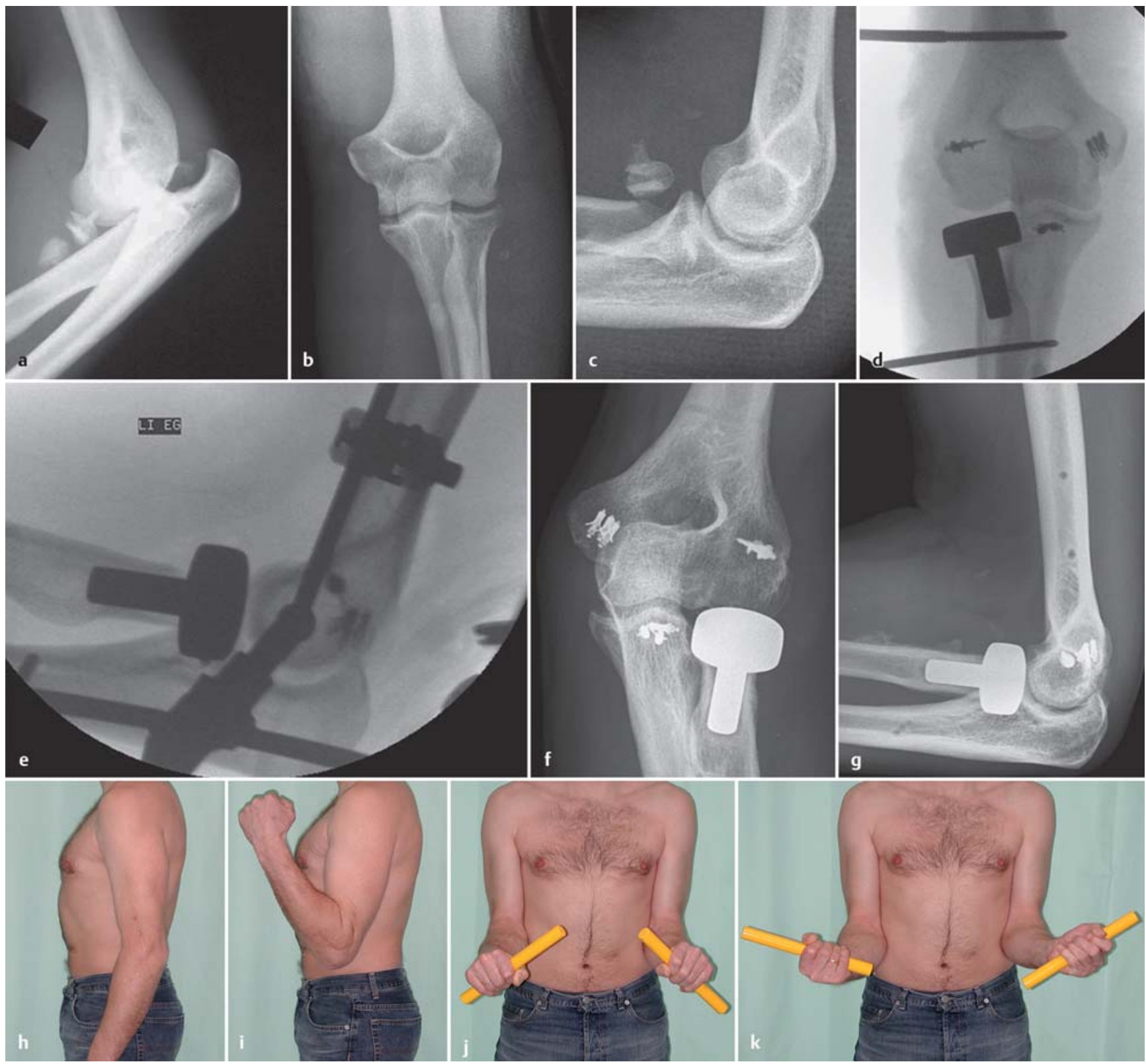

Abb. $\mathbf{8}$ a bis k 48-jähriger Patient. Sturz beim Tennisspielen. Luxationsfraktur des linken Ellbogengelenks. a-c Nativröntgen - luxierter Zustand, Zustand nach Reposition (Radiuskopffraktur, Abbruch des Processus coronoideus ulnae); d, e intraoperative Bildwandleraufnahme (Radiuskopfprothese, Bandrekonstruktion lateral und medial, Bewegungs-Fixateur externe); $\mathbf{f}-\mathbf{k}$ Nativröntgen und funktionelles Ergebnis nach 3 Jahren.

griffels (des abgespreizten Daumens). Eine Fehlrotation der Prothese muss unbedingt vermieden werden, da es sonst bei Unterarmrotation zu einer Luxation der Prothese oder zu asymmetrischer Belastung des Gelenkknorpels am Capitulum kommt. Weiterhin konnte gezeigt werden, dass ein Ausmaß der primären Resektion oder eines Knochenverlusts von mehr als $15 \mathrm{~mm}$ am proximalen Radius zu einer erheblichen Zunahme der Mikrobewegungen zwischen Schaft und Knochen und damit zu einer potenziellen frühzeitigen Auslockerung führt [28].

Ein weiterer sehr entscheidender Faktor für das Langzeit-Outcome einer Radius- kopfprothese ist die optimale Größe und Länge der Prothese, was insbesondere bei gleichzeitiger Instabilität des Ellbogengelenks schwierig sein kann. Der Durchmesser der Radiuskopfprothese wird i.d.R. ca. $2 \mathrm{~mm}$ geringer genommen als es dem gemessenen Durchmesser des entfernten nativen Radiuskopfs entspricht. Die korrekte Länge der Radiuskopfprothese wird intraoperativ durch die Incisura radialis der Elle definiert, wobei bei gleichzeitigem Vorliegen einer Membranverletzung zuerst auf eine seitengleiche korrekte Stellung im distalen Radioulnargelenk geachtet werden muss. Dann darf der Prothesenkopf der Radiuskopfprothese die Inzisur nach proximal nicht überragen. Intraoperativ dient die laterale Gelenkkante des Processus coronoideus als Landmarke. Der Prothesenkopf darf diesen nach proximal nicht überragen oder sollte sogar bis zu $2 \mathrm{~mm}$ weiter distal positioniert sein. Da die individuellen anatomischen Unterschiede in diesem Bereich groß sind, sollte präoperativ ein Röntgen des Handgelenks und des Ellbogengelenks der gesunden Seite als Referenz durchgeführt werden $[29,30]$. Weiterhin sollte intraoperativ immer nach Setzen der Probeprothese eine Bildwandleruntersuchung vorgenommen werden und exakt auf die richtige Länge der Prothese in Relation zur lateralen Gelenkkante der Elle 
geachtet werden. Weiterhin muss der humeroulnare Gelenkspalt symmetrisch sein und parallele Gelenkflächen aufweisen. Nach Implantation der definitiven Radiuskopfprothese sollte am Rückweg der laterale Bandapparat stabil rekonstruiert werden. Ist auch der mediale Bandapparat defekt, sind wir bei implantierter Radiuskopfprothese dazu übergegangen, den medialen Seitenbandapparat ebenfalls immer zu rekonstruieren, um die Kraftspitzen am radialen Gelenkabschnitt zu reduzieren.

Wichtig für ein gutes Langzeitergebnis nach Implantation einer Radiuskopfprothese ist die Vermeidung einer Überlänge der Prothese, eines zu großen Prothesenkopfs sowie bei Langschaftprothesen die korrekte Ausrichtung der Prothese unter Berücksichtigung der natürlichen Schaft-Hals-Krümmung am proximalen Radius.

\section{Ergebnisse}

Die mittelfristigen Ergebnisse der modernen modularen Radiuskopfprothesen sind vielversprechend. Unter Berücksichtigung der wichtigen operationstechnischen Details, wie korrekte Größe und Länge und stabile Rekonstruktion der Bänder, lassen sich in der überwiegenden Mehrzahl der Fälle sehr gute und gute Ergebnisse erzielen (Abb.8). Obwohl auch nach sekundär fehlgeschlagener Osteosynthese bei noch gut erhaltenem Gelenkknorpel am Capitulum radialis humeri noch gute Ergebnisse erzielt werden können, zeigt die Erfahrung, dass die primäre Radiuskopfprothese bei richtiger Indikation bessere Ergebnisse bringt [31].

Knorpelschäden am Capitulum radialis humeri können vom initalen Trauma herrühren oder im Verlauf durch die Radiuskopfprothese ausgelöst sein. Schmerzen und Knorpelveränderungen sind umso rascher progredient, je unanatomischer die Implantation der Prothese erfolgte. Hier kann in Zukunft evtl. die Implantation einer zusätzlichen Teilprothese des Capitulums am distalen Humerus eine Lösungsmöglichkeit darstellen. Erste Erfahrungen damit sind vielversprechend [32]. Die Datenlage ist aber für eine aussagekräftige Beurteilung noch nicht ausreichend. Ansonsten kommt in weiterer Folge, wenn keine Instabilität mehr besteht, die Radiuskopfresektion und beim Fortschreiten der Beschwerden die Ellbogengelenktotalendoprothese infrage.

\section{Schlussfolgerung}

Die Endoprothetik spielt heute in der Versorgung von komplexen Frakturen am Ellbogengelenk eine immer bedeutendere Rolle. Ob in Form der Radiuskopfprothese, als Hemiprothese oder als Totalendoprothese des Ellbogengelenks stellt sie sowohl in der primären Frakturversorgung wie auch sekundär bei Zustand nach Traumen mit fehlverheilten Frakturen, Pseudarthrosen, Instabilitäten und posttraumatischer Arthrose eine sehr vielversprechende Versorgungsoption dar.

Interessenkonflikt: Nein.

\section{Literatur}

${ }^{1}$ Robinson CM, Hill RM, Jacobs N et al. Adult distal humeral metaphyseal fractures: epidemiology and results of treatment. J Orthop Trauma 2003; 17: 38-47

2 Letsch R, Schmit-Neuerburg KP, Stürmer KM et al. Intraarticular fractures of the distal humerus. Surgical treatment and results. Clin Orthop Relat Res 1989; 241: 238-244

3 JupiterJB, NeffU, Regazzoni Pet al. Unicondylar fractures of the distal humerus: an operative approach. J Orthop Trauma 1988; 2: 102-109

4 O'Driscoll SW. Supracondylar fractures of the elbow: open reduction, internal fixation. Hand Clin 2004; 20: 465-474

5 Södergard J, Sandelin J, Bostman O. Mechanical failures of internal fixation in $T$ and Y fractures of the distal humerus. J Trauma 1992; 33: 687-690

6 Korner J, Lill H, Müller LP et al. Distal humerus fractures in elderly patients: results after open reduction and internal fixation. Osteoporosis Int 2005; 16 (Suppl. 2): S73-S79

7 Claessen FM, Braun Y, Peters RM et al. Plate and Screw Fixation of Bicolumnar Distal Humerus Fractures: Factors Associated With Loosening or Breakage of Implants or Nonunion. J Hand Surg Am 2015; 40: 2045-2051

8 Cobb TK, Morrey BF. Total elbow arthroplasty as primary treatment for distal humeral fractures in elderly patients. J Bone Joint Surg Am 1997; 79: 826-832

${ }^{9}$ Kamineni S, Morrey BF. Distal humeral fractures treated with noncustom total elbow replacement. J Bone Joint Surg Am 2004; 86A: 940-947

10 Athwal GS, Goetz TJ, PollockJW et al. Prosthetic replacement for distal humerus fractures. Orthop Clin North Am 2008; 39: 201-212

11 Mighell MA, Frankle MA, Mulieri PJ. Elbow arthroplasty for distal humeral fractures Technique, pearls and pitfalls. Oper Tech Orthop 2010; 20: 38-47

12 Ducrot G, Ehlinger M, Adam P et al. Complex fractures of the distal humerus in the elderly: is primary total elbow arthroplasty a valid treatment alternative? A series of 20 cases. Orthop Traumatol Surg Res 2013; 99: 10-20

13 Müller LP, Wegmann K, Burkhart KJ. Frakturendoprothetik der distalen Humerusfraktur. Unfallchirurg 2013; 116: 708-715

14 Harmer LS, Sanchez-Sotelo J. Total elbow arthroplasty for distal humerus fractures. Hand Clin 2015; 31: 605-614

15 Pogliacomi F, Schiavi P, Defilippo M. Total elbow arthroplasty following complex fractures of the distal humerus: results in patients over 65 years of age. Acta Biomed 2016; 87: 148-155

16 McKee MD, Veillette CJ, Hall JA et al. A multicenter, prospective, randomized, controlled trial of open reduction - internal fixation ver- sus total elbow arthroplasty for displaced intra-articular distal humeral fractures in elderly patients. J Shoulder Elbow Surg 2009; 18: $3-12$

17 Egol KA, Tsai P, Vazques 0 et al. Comparison of functional outcomes of total elbow arthroplasty vs plate fixation for distal humerus fractures in osteoporotic elbows. Am J Orthop 2011; 40: 67-71

18 Nestorson J, Ekholm C, Etzner $M$ et al. Hemiarthroplasty for irreversible distal humeral fractures: medium-term follow-up of 42 patients. Bone Joint J 2015; 97-B: 1377-1384

19 Burkhart KJ, Nijs S, Mattyasovszky SG et al. Distal humerus hemiarthroplasty of the elbow for comminuted distal humeral fracture in the elderly patient. J Trauma 2011; 71: 635-642

20 Smith GC, Hughes IS. Unreconstructable acute distal humeral fractures and their sequelae treated with distal humeral hemiarthroplasty: a two-year to eleven-year follow-up. J Shoulder Elbow Surg 2013 : 22: 1710-1723

21 Hohman DW, Nodzo SR, Qvick LM et al. Hemiarthroplasty of distal humerus for acute and chronic complex intra-articular injuries. J Shoulder Elbow Surg 2014; 23: 265-272

22 Steinmann SP. Hemiarthroplasty of the ulnohumeral and radiocapitellar joints. Hand Clin 2011: 27: 229-232

23 Studer A, Athwal GS, MacDermid JC et al. The lateral para-olecranon approach for total elbow arthroplasty. J Hand Surg Am 2013; 38: 2219-2226

${ }^{24}$ McKee MD, Pugh DM, Richards RR et al. Effect of humeral condylar resection on strength and functional outcome after semiconstrained total elbow arthroplasty. J Bone Joint Surg Am 2003; 85-A: 802-807

25 Githens M, Yao J, Sox AH et al. Open reduction and internal fixation versus total elbow arthroplasty for the treatment of geriatric distal humerus fractures: a systematic review and meta-analysis. J Orthop Trauma 2014; 28: 481-488

26 Prasad N, Dent C. Outcome of total elbow replacement for distal humeral fractures in the elderly. A comparison of primary surgery and surgery after failed internal fixation or conservative treatment. J Bone Joint Surg $\mathrm{Br}$ 2008; 90: 343-348

27 Itamura J, Roidis N, Mirzayan $R$ et al. Radial head fractures: MRI evaluation of associated injuries. J Shoulder Elbow Surg 2005; 14: 421-424

28 Shukla DR, Fitzsimmons JS, An K et al. Effect of stem length on prosthetic radial head micromotion. J Shoulder Elbow Surg 2012; 21: 1559-1564

29 Doornberg JN, Linzel DS, Zurakowski D et al. Reference points for radial head prosthesis size. J Hand Surg Am 2006; 31: 53-57

30 King G. Fractures of the Radial Head. In: Wolfe SW, Hotchkiss RN, Pederson WC, Kozin SH, eds. Green's Operative Hand Surgery. 6th ed. Philadelphia: Elsevier; 2011: 783-819

31 King GJ. Management of comminuted radial head fractures with replacement arthroplasty. Hand Clin 2004; 20: 429-441

32 Kepler CK, Kummer JL, Lorich DG et al. Radiocapitellar prosthetic arthroplasty for capitellar nonunion. J Shoulder Elbow Surg 2010; 19: e13-e17

\section{Prim. Dr. med. Michael Plecko}

\section{Ärztlicher Leiter}

Unfallkrankenhaus der AUVA, Graz

Lehrkrankenhaus der MEDUNI, Graz

Göstingerstraße 24

8021 Graz

Österreich

michael.plecko@auva.at 